\title{
RESULTADOS CLÍNICOS Y PERINATALES DE LOS EMBARAZOS CON HIPERTENSIÓN ARTERIAL EN UN HOSPITAL DE REFERENCIA DE LA VIII REGIÓN DE CHILE
}

\author{
Patricio Bertoglia F. 1,2, Alba Rivas P. ${ }^{2 a}$, Patricia Navarrete R. ${ }^{2 a}$, Luis Castro M. ${ }^{3}$, \\ Jesenia Acurio J. ${ }^{2 b}$, Carlos Escudero O. ${ }^{2}, \mathrm{PhD}$. \\ 1 Departamento de Ginecología y Obstetricia, Hospital Herminda Martín; ${ }^{2}$ Laboratorio de Fisiología Vascular, Depar- \\ tamento de Ciencias Básicas, Universidad del Bío-Bío, Chillán, Chile; ${ }^{3}$ Centro de Salud de San José de Chuchunco, \\ Santiago, Chile.
}

$a_{\text {Bachiller en Ciencias Naturales y Exactas. }}{ }^{b}$ Técnico en Laboratorio Clínico y Banco de Sangre.

\section{RESUMEN}

Objetivo: Conocer los resultados clínicos, bioquímicos y perinatales asociados al síndrome de hipertensión del embarazo (SHE) en el Hospital Herminda Martín de Chillán. Métodos: Se realizó un estudio retrospectivo de registros clínicos $(n=416)$ con diagnóstico de SHE en el periodo 2006 a 2008. Los registros disponibles fueron divididos en tres grupos de acuerdo al nivel de presión arterial: Grupo I $(n=124)<140 / 90$ mmHg; Grupo II $(n=98) \geq 140-159 / \geq 90-109 \mathrm{mmHg}$ y Grupo III $(n=41) \geq 160 / 110 \mathrm{mmHg}$. Adicionalmente, un subgrupo $(n=85)$ fue dividido considerando el percentil de distribución del nivel de ácido úrico materno en: SHE con niveles bajos (<p25), medios (p25-p75) o altos (>p75). Se analizaron y compararon los grupos estudiados y se correlacionó las variables estudiadas con los resultados perinatales. Resultados: La prevalencia de SHE fue de 3,8\%. Las mujeres del grupo II y III muestran peores resultados clínicos y neonatales que las mujeres del grupo I. El índice de masa corporal (IMC), la presión arterial materna y el nivel de ácido úrico están relacionados negativamente con la antropometría neonatal. Además, la antropometría neonatal fue menor en las mujeres con niveles más altos de ácido úrico, situación que no obedece a la severidad de la hipertensión o el IMC materno. Conclusión: La presencia de SHE esta asociada a mayor morbilidad materna y neonatal. Este estudio permitió detectar deficiencias (e.i., falta de cumplimiento en criterio diagnóstico) y hacer recomendaciones sobre probables marcadores de riesgo perinatal (e.i., nivel de ácido úrico).

\section{PALABRAS CLAVES: Hipertensión del embarazo, resultados clínicos y perinatales}

\section{SUMMARY}

Aims: To know the clinical and biochemical spectra and perinatal outcomes associated with syndrome of hypertension in pregnancy (HP) in the Herminda Martín Hospital from Chillán. Methods: It is a retrospective study using clinical records with HP diagnosis $(\mathrm{N}=416)$ during 2006 to 2008. The available records were divided in three different groups according with arterial blood pressure: Group I $(n=124)<140 / 90 \mathrm{mmHg}$; Group II $(n=98) \geq 140-159 / \geq 90-109 \mathrm{mmHg}$ and Group III $(n=41) \geq 160 / 110 \mathrm{mmHg}$. Additionally, a subset $(n=85)$ was divided considering the percentile of the uric acid level in the mother as: HP with low $(<p 25$ th), middle (p25-75th) and high ( $>$ p75th) uric acid levels. The studied groups were analyzed and compared each other and quantitative variables were correlated with perinatal outcomes. Results: The prevalence of $\mathrm{HP}$ was $3.8 \%$. Women in the groups II and III showed worse clinical and neonatal outcomes compared with 
women in the group I. Body mass index (BMI), maternal blood pressure and uric acid levels were negatively correlated with neonatal anthropometry. Moreover, neonatal anthropometry was lower in women with high uric acid levels, a situation that was independent of maternal hypertension or BMI. Conclusion: Hypertension in pregnancy was associated with high maternal and neonatal morbidity. This study allowed identifies some weaknesses (e.i., lack of compliance in diagnosis criteria) as well as suggest the potential role of perinatal risk markers (e.i., uric acid levels).

\section{KEY WORDS: Hypertension in pregnancy, clinical and perinatal outcomes}

\section{INTRODUCCIÓN}

El síndrome hipertensivo del embarazo (SHE) es una patología característica del embarazo humano que provoca un daño multisistémico y que incluye varias entidades patológicas (1). Así, el SHE se clasifica en: hipertensión inducida por el embarazo (hipertensión sin proteinuria, preeclampsia y eclampsia) e hipertensión crónica (hipertensión esencial e hipertensión secundaria) (2). Además, la preeclampsia puede ser subdividida en preeclampsia moderada (presión arterial $\geq 140 / 90 \mathrm{mmHg}$, proteinuria $\geq 300 \mathrm{mg} / 24 \mathrm{~h}$ ) o preeclampsia severa (presión arterial $\geq 160 / 110 \mathrm{mmHg}$, proteinuria $\geq 5$ g/24 h) (3-5). Estos criterios son generalmente utilizados en América, sin embargo existen otras recomendaciones que difieren sustancialmente (6).

La prevalencia de preeclampsia varía entre el 6 y $30 \%$ de los embarazos a nivel mundial (7-10). En Chile, fluctúa entre 7 y $10 \%$, siendo una importante causa de mortalidad materna, fetal y neonatal $(11,12)$. De hecho, el SHE con sus variantes clínicas, ocupan del tercer al sexto lugar entre las principales causas de muerte materna en Chile (13). Además, a nivel mundial $(8)$ y nacional $(12,14)$, la preeclampsia aumenta el riesgo de retardo de crecimiento intrauterino (RCIU), bajo peso al nacer, prematurez y morbimortalidad perinatal comparadas con el embarazo normal.

En la región del Bío-Bío (VIII Región, Chile) existen pocos reportes (14-16) enfocados en el estudio de las características clínicas y complicaciones del SHE en la salud materna y fetal. Durante el 2005 de acuerdo a las estadísticas del Ministerio de Salud, del total de egresos hospitalarios atribuidos a enfermedades del embarazo, parto y puerperio en el servicio de salud de Ñuble, el $50 \%$ fueron atendidos en el Hospital Herminda Martín de Chillán (17), sin embargo, al momento no existen reportes sobre el análisis de los embarazos complicados con SHE de este centro de referencia de la VIII Región. Por ello, el objetivo de este estudio es conocer las características clínicas y bioquímicas, y las complicaciones del recién nacido asociadas a SHE en el Hospital Herminda Martín de Chillán.

\section{PACIENTES Y MÉTODOS}

Estudio retrospectivo, de revisión de fichas clínicas de las mujeres diagnosticadas con SHE en el servicio de Alto Riesgo Obstétrico (ARO) del Departamento de Ginecología y Obstetricia del Hospital Clínico Herminda Martín de Chillán, entre enero de 2006 y diciembre de 2008. El universo de estudio comprendió las fichas clínicas de pacientes que ingresaron a ARO y que posteriormente fueron localizadas en el Departamento de Estadística del Hospital.

Se investigaron datos clínicos y bioquímicos de las mujeres, así como datos antropométricos de las madres y los recién nacidos. Se evaluó la presencia de preeclampsia moderada o severa después de la semana 20 de gestación de acuerdo a los criterios antes enunciados (3-5). Sin embargo, dado que en el $86 \%$ de las mujeres con sospecha de preeclampsia, de acuerdo al rango de presión arterial, no disponían de un registro de proteinuria, se utilizó únicamente el criterio de presión arterial para clasificar los registros en tres grupos de estudio: Grupo I $(n=124)<140 / 90 \mathrm{~mm} \mathrm{Hg}$; Grupo II $(n=98) \geq 140$ $159 / \geq 90-109 \mathrm{mmHg}$ y Grupo III $(n=41) \geq 160 / 110$ $\mathrm{mmHg}$.

Adicionalmente, se recogieron datos de creatininemia, recuento de plaquetas, niveles de enzimas hepáticas (GOT y GPT), presencia de anemia hemolítica microangiopática (aumento LDH) $(12,18)$. Además, se hizo énfasis en el registro de los niveles plasmáticos maternos de ácido úrico $(n=85), y$ se clasificaron en SHE con niveles bajos (Bajo, < percentil 25, de 2,6 a 3,7 mg/dL, $\mathrm{n}=26$ ), niveles medios (Medio, entre el percentil 25 a 75, de 3,8 a 5,4 $\mathrm{mg} / \mathrm{dL}$ ) o niveles altos de ácido úrico (Alto, superior al percentil $75, \geq 5,8 \mathrm{mg} / \mathrm{dL}$ ).

Se definió como retardo de crecimiento intrauterino (RCIU) al peso del recién nacido por debajo del percentil 10 de acuerdo a la edad gestacional (19). A su vez, se reporta como otras complicaciones del recién nacido, la presencia de sufrimiento fetal agudo $(n=13)$, hiperbilirrubinemia $(n=8)$, síndrome de distrés respiratorio $(n=5)$, bradicardia fetal $(n=5)$, macrocefalia $(n=2)$ y fractura clavicular $(n=2)$. 
Las variables cualitativas se expresan en porcentaje respecto al grupo analizado, mientras que las variables cuantitativas se expresan en promedio \pm error estándar (EE). Las variables cualitativas se compararon utilizando la prueba de Chi2 y las cuantitativas fueron comparadas utilizando un análisis de varianza paramétrico (ANOVA). Además se compararon los diferentes grupos en pares utilizando un análisis múltiple de Dunn. Adicionalmente, utilizando el grupo completo de registros se determinó la correlación (Pearson, con un intervalo de confianza de 95\%) del IMC, la presión arterial, conteo plaquetario y niveles de ácido úrico con los indicadores clínicos y bioquímicos analizados en este estudio. Se fijó un valor $p<0,05$ para determinar diferencias estadísticas.

\section{RESULTADOS}

En el período estudiado hubo 416 pacientes con SHE y 11.039 partos, determinando una prevalencia de $3,8 \%$. De las fichas clínicas solo fue posible ubicar a 263 pacientes (63\%), de las cuales el 53\% mostró sospecha de preeclampsia de acuerdo al nivel de presión arterial ( $\geq 140 / 90 \mathrm{mmHg}$ ). Únicamente fue posible identificar algún registro de proteinuria en el $40 \%$ de estas mujeres, de las cuales cumplían criterios diagnósticos de preeclampsia ( $\geq 300 \mathrm{mg} / 24 \mathrm{~h}$ ) sólo el 14\% (rango identificado en estos registros entre 300 y 1.000 mg/24h). Las características clínicas de las mujeres estudiadas se presentan en la Tabla I.

La tasa de mortalidad fetal en este grupo estudiado fue de $3,4 \%$, de RCIU de $6,1 \%$, de parto prematuro (<37 semanas de gestación) de 6,5\%, y de otras complicaciones del recién nacido (ver Métodos) de 13\%. Los datos del parto y del recién nacido se presentan en la Tabla II.

Se encontró en los registros analizados datos de GOT y GPT $(n=87)$, bilirrubinemia $(n=91)$, hematocrito $(n=162)$, LDH $(n=26)$, plaquetas $(n=146)$, creatinina $(n=96)$ y ácido úrico $(n=85)$. En la Tabla III se muestran los niveles de los marcadores bioquímicos. De acuerdo a los datos recolectados no existieron casos de síndrome de HELLP.

Se presenta además la correlación entre índice de masa corporal (Tabla IV), presión arterial (Tabla V) y plaquetas y ácido úrico (Tabla VI), con las distintas variables analizadas en el total de registros estudiados.

En la Tabla VII se presenta las características clínicas maternas y del recién nacido en los embarazos con diagnóstico de SHE que presentaron niveles de ácido úrico Bajo, Medio o Alto.

Tabla I

\section{CARACTERISTICAS DE LAS MUJERES CON SINDROME HIPERTENSIVO DEL EMBARAZO EN EL HOSPITAL HERMINDA MARTIN DE CHILLAN}

\begin{tabular}{|c|c|c|c|c|c|}
\hline Característica & $\begin{array}{l}\text { Grupo I } \\
(n=124)\end{array}$ & $\begin{array}{c}\text { Grupo II } \\
(n=98)\end{array}$ & $\begin{array}{c}\text { Grupo III } \\
(n=41)\end{array}$ & $\begin{array}{c}\text { TOTAL } \\
(n=263)\end{array}$ & $p$ \\
\hline Edad (años \pm E.E) & $26,9 \pm 0,6$ & $28,5 \pm 0,7$ & $29,4 \pm 1,2$ & $28 \pm 0,4$ & NS \\
\hline Peso inicial $(\mathrm{kg} \pm \mathrm{E} . \mathrm{E})$ & $68,7 \pm 1,3$ & $73,4 \pm 1,7$ & $71,8 \pm 2,9$ & $71 \pm 1,0$ & NS \\
\hline Peso final (kg \pm E.E) & $82,6 \pm 1,5$ & $87,4 \pm 1,7^{\star}$ & $88,9 \pm 2,7$ & $85 \pm 1,1$ & 0,04 \\
\hline Talla $(\mathrm{cm} \pm \mathrm{E} . \mathrm{E})$ & $153 \pm 2$ & $153 \pm 2$ & $153 \pm 4$ & $153 \pm 1,4$ & NS \\
\hline IMC (kg/m2 \pm E.E) & $33,9 \pm 0,7$ & $35,8 \pm 0,6^{\star}$ & $35,9 \pm 1,2$ & $34,9 \pm 0,5$ & 0,02 \\
\hline Sobrepeso (\%) & 17 & 22 & 24 & 23 & NS \\
\hline Obesidad (\%) & 37 & $59^{\star}$ & $61^{*}$ & 52 & 0,001 \\
\hline Antecedente hipertensión (\%) & 20 & 26 & $41^{*} \ddagger$ & 26 & 0,05 \\
\hline Ant. hipertensión familiar (\%) & 22 & 26 & 24 & 20 & NS \\
\hline Preeclampsia antecedentes (\%) & 4 & 8 & 5 & 6 & NS \\
\hline Nuliparas (\%) & 40 & 36 & 44 & 40 & NS \\
\hline Gestas (\# \pm E.E) & $1,7 \pm 0,1$ & $2,1 \pm 0,2$ & $2,1 \pm 0,3$ & $1,1 \pm 0,1$ & NS \\
\hline Última gesta (meses \pm E.E) & $79,1 \pm 6,8$ & $91,0 \pm 7,1$ & $106,4 \pm 14,0$ & $87,7 \pm 4,6$ & NS \\
\hline
\end{tabular}

Valores expresados en promedio \pm error estándar (E.E) o porcentaje (\%) respecto a su propio grupo. Índice de masa corporal (IMC). Grupo I, presión arterial <140/90 mm Hg, Grupo II, presión arterial entre 140/90 y 159/109 mm Hg y Grupo III presión arterial $>160 / 110 \mathrm{~mm} \mathrm{Hg.}{ }^{*} p<0,05$ vs Grupo I. $\neq p<0,05$ vs Grupo II. NS: no significativo, 
Tabla II

CARACTERISTICAS DE LOS RECIEN NACIDOS DE LAS MUJERES CON SINDROME HIPERTENSIVO DEL EMBARAZO EN EL HOSPITAL HERMINDA MARTIN DE CHILLAN

\begin{tabular}{|c|c|c|c|c|c|}
\hline Característica & $\begin{array}{l}\text { Grupo I } \\
(n=124)\end{array}$ & $\begin{array}{c}\text { Grupo II } \\
(n=98)\end{array}$ & $\begin{array}{c}\text { Grupo III } \\
(n=41)\end{array}$ & $\begin{array}{l}\text { TOTAL } \\
(\mathrm{n}=263)\end{array}$ & $p$ \\
\hline EG parto (sem \pm E.E) & $38,5 \pm 0,3$ & $38,7 \pm 0,2$ & $36,9 \pm 0,4^{*}$ & $38,3 \pm 0,1$ & 0,0001 \\
\hline Parto prematuro (<35 semanas, \%) & 2,4 & 3,1 & $17,1^{*} \ddagger$ & 4,9 & 0,05 \\
\hline Días hospitalización (días \pm E.E) & $2,5 \pm 0,1$ & $3,4 \pm 0,3$ & $3,7 \pm 0,4^{*}$ & $3,1 \pm 0,2$ & 0,003 \\
\hline Peso RN (g \pm E.E) & $3434 \pm 54,2$ & $3500 \pm 62,0$ & $2977 \pm 109^{\star} \ddagger$ & $3389 \pm 39,9$ & $<0,0001$ \\
\hline Percentil peso $(g \pm E . E)$ & $60,3 \pm 2,7$ & $66,2 \pm 2,7$ & $54,7 \pm 4,4 \ddagger$ & $61,6 \pm 1,8$ & 0,04 \\
\hline Talla (cm \pm E.E) & $49,1 \pm 0,2$ & $49,4 \pm 0,3$ & $47,8 \pm 0,4^{\star} \ddagger$ & $49,0 \pm 0,2$ & 0,04 \\
\hline Percentil talla $(\mathrm{cm} \pm \mathrm{E} . \mathrm{E})$ & $53,8 \pm 2,8$ & $59,8 \pm 2,9$ & $51,9 \pm 4,4$ & $55,8 \pm 1,9$ & NS \\
\hline RCIU (\%) & 7,2 & 2,0 & $12,2 \ddagger$ & 6,1 & 0,05 \\
\hline Apgar 1 min $( \pm$ E.E) & $8,5 \pm 0,1$ & $8,1 \pm 0,1$ & $8,1 \pm 0,3^{*}$ & $8,3 \pm 0,1$ & 0,01 \\
\hline Apgar 5 min $( \pm$ E.E) & $9,2 \pm 0,1$ & $9,0 \pm 0,1$ & $9,1 \pm 0,1$ & $9,2 \pm 0,05$ & NS \\
\hline Muerte fetal (\%) & 5 & 3 & $0^{*}$ & 3,4 & 0,05 \\
\hline Otras complicaciones RN (\%) & 13 & 11 & 17 & 13 & NS \\
\hline
\end{tabular}

Valores expresados en promedio \pm error estándar (E.E) o porcentaje (\%) respecto a su propio grupo. Edad gestacional de parto (EG parto). Recién nacido, RN. Retardo de crecimiento intrauterino, RCIU. Otras complicaciones del recién nacido incluyen la presencia de sufrimiento fetal agudo $(n=13)$, hiperbilirrubinemia $(n=8)$, síndrome de distrés respiratorio $(n=5)$, bradicardia fetal $(n=5)$, macrocefalia $(n=2)$ y fractura clavicular $(n=2)$. Grupo I, presión arterial $<140 / 90 \mathrm{~mm} \mathrm{Hg}$, Grupo II, presión arterial entre $140 / 90$ y $159 / 109 \mathrm{~mm} \mathrm{Hg}$ y Grupo III presión arterial $>160 / 110 \mathrm{~mm} \mathrm{Hg}$. ${ }^{*} p<0,05$ vs Grupo I. łp $<0,05$ vs Grupo II. No estadísticamente significativo, NS

Tabla III

MARCADORES DE SEVERIDAD DEL SINDROME HIPERTENSIVO DEL EMBARAZO EN EL HOSPITAL HERMINDA MARTIN DE CHILLAN

\begin{tabular}{|c|c|c|c|c|c|}
\hline Característica & $\begin{array}{l}\text { Grupo I } \\
(n=124)\end{array}$ & $\begin{array}{c}\text { Grupo II } \\
(n=98)\end{array}$ & $\begin{array}{c}\text { Grupo III } \\
(n=41)\end{array}$ & $\begin{array}{c}\text { TOTAL } \\
(n=263)\end{array}$ & $\mathrm{p}$ \\
\hline $\mathrm{PAS}(\mathrm{mm} \mathrm{Hg} \pm \mathrm{E} . \mathrm{E})$ & $124,0 \pm 0,7$ & $145,0 \pm 0,5^{\star}$ & $170,5 \pm 1,9^{\star}$ & $139 \pm 1,1$ & $<0,0001$ \\
\hline $\mathrm{PAD}(\mathrm{mm} \mathrm{Hg} \pm \mathrm{E} . \mathrm{E})$ & $78,6 \pm 1,2$ & $94,0 \pm 1,2^{\star}$ & $110,8 \pm 1,6^{\star}$ & $89,2 \pm 1,1$ & $<0,0001$ \\
\hline GOT (UI \pm E.E) & $29,3 \pm 3,4$ & $31,7 \pm 4,8$ & $28,9 \pm 2,3$ & $30,4 \pm 2,5$ & NS \\
\hline GPT (UI \pm E.E) & $30,3 \pm 2,2$ & $30,2 \pm 2,8$ & $29,8 \pm 2,0$ & $30,2 \pm 1,5$ & NS \\
\hline Bilirrubina (UI \pm E.E) & $0,31 \pm 0,02$ & $0,30 \pm 0,02$ & $0,38 \pm 0,06$ & $0,32 \pm 0,02$ & NS \\
\hline Hematocrito $(\% \pm$ E.E) & $35,7 \pm 0,4$ & $34,8 \pm 0,6$ & $34,9 \pm 0,9$ & $35,0 \pm 0,4$ & NS \\
\hline $\mathrm{LDH}(\mathrm{UI} \pm \mathrm{E} . \mathrm{E})$ & $205 \pm 16$ & $264 \pm 48$ & $221 \pm 19$ & $256 \pm 29$ & NS \\
\hline Plaquetas $\left(\# 10^{3} \pm E . E\right)$ & $237 \pm 8,9$ & $210 \pm 8,3$ & $194 \pm 8,9^{\star}$ & $218 \pm 5,3$ & 0,01 \\
\hline Creatinina $(m g \pm E . E$ & $0,7 \pm 0,03$ & $0,7 \pm 0,04$ & $0,8 \pm 0,05$ & $0,7 \pm 0,02$ & NS \\
\hline Acido úrico $(\mathrm{mg} \pm \mathrm{E} . \mathrm{E})$ & $4,3 \pm 0,3$ & $5,2 \pm 0,5$ & $5,1 \pm 0,3$ & $4,9 \pm 0,3$ & NS \\
\hline
\end{tabular}

Valores expresados en promedio \pm error estándar (E.E). Presión arterial sistólica, PAS. Presión arterial diastólica, PAD. Transaminasa oxalacética, GOT. Transaminasa pirúvica, GTP. Lactato deshidrogenasa, LDH. Unidades internacionales (UI). Grupo I, presión arterial <140/90 mm Hg, Grupo II, presión arterial entre 140/90 y 159/109 mm Hg y Grupo III presión arterial $>160 / 110 \mathrm{~mm} \mathrm{Hg}$. ${ }^{*} p<0,05$ vs Grupo I. No estadísticamente significativo, NS. 
Tabla IV

RELACION DEL INDICE DE MASA CORPORAL CON VARIABLES CLINICAS MATERNAS Y NEONATALES EN LAS MUJERES CON SINDROME HIPERTENSIVO DEL EMBARAZO EN EL HOSPITAL HERMINDA MARTIN DE CHILLAN

\begin{tabular}{lcccc}
\hline Variable & $r$ & $95 \%$ IC & $p$ & $n$ \\
\hline PAS & 0,14 & 0,01 a 0,26 & 0,03 & 228 \\
PAD & 0,12 & $-0,004$ a 0,25 & 0,06 & 228 \\
Peso RN & 0,20 & 0,07 a 0,33 & 0,002 & 216 \\
Percentil peso RN & 0,24 & 0,12 a 0,37 & 0,002 & 216 \\
Talla RN & 0,16 & 0,02 a 0,29 & 0,01 & 213 \\
Percentil talla RN & 0,18 & 0,04 a 0,31 & 0,007 & 213 \\
EG parto & 0,11 & $-0,02$ a 0,23 & 0,11 & 225 \\
Plaquetas & 0,13 & $-0,04$ a 0,29 & 0,13 & 129 \\
Acido Úrico & $-0,23$ & $-0,44$ a - 0,018 & 0,03 & 78
\end{tabular}

Correlación de Pearson, r. Intervalo de confianza del 95\%, 95\% IC. Presión arterial sistólica, PAS. Presión arterial diastólica, PAD. Recién nacido, RN. Edad gestacional, EG.

Tabla V

RELACION DE LA PRESION ARTERIAL SISTOLICA Y DIASTOLICA CON VARIABLES CLINICAS MATERNAS Y NEONATALES EN LAS MUJERES CON SINDROME HIPERTENSIVO DEL EMBARAZO EN EL HOSPITAL HERMINDA MARTIN DE CHILLAN

\begin{tabular}{|c|c|c|c|c|}
\hline Variable & $r$ & $95 \%$ IC & $p$ & $\mathrm{n}$ \\
\hline \multicolumn{5}{|c|}{ Presión arterial sistólica } \\
\hline PAD & 0,71 & 0,64 a 0,76 & $<0,0001$ & 263 \\
\hline Peso RN & $-0,17$ & $-0,29$ a $-0,05$ & 0,007 & 247 \\
\hline Percentil peso RN & 0,02 & $-0,01$ a 0,15 & 0,67 & 247 \\
\hline Talla RN & 0,12 & $-0,24$ a 0,003 & 0,06 & 245 \\
\hline Percentil talla RN & 0,05 & $-0,08$ a 0,17 & 0,47 & 245 \\
\hline EG parto & $-0,16$ & $-0,28$ a $-0,03$ & 0,009 & 257 \\
\hline Plaquetas & $-0,22$ & $-0,37$ a $-0,06$ & 0,006 & 145 \\
\hline Acido Úrico & 0,06 & $-0,14$ a 0,27 & 0,54 & 85 \\
\hline \multicolumn{5}{|c|}{ Presión arterial diastólica } \\
\hline Peso RN & $-0,16$ & $-0,28$ a $-0,03$ & 0,01 & 247 \\
\hline Percentil peso RN & $-0,03$ & $-0,02$ a 0,08 & 0,57 & 247 \\
\hline Talla RN & $-0,10$ & $-0,22$ a 0,02 & 0,09 & 245 \\
\hline Percentil talla $\mathrm{RN}$ & 0,02 & $-0,11$ a 0,14 & 0,77 & 245 \\
\hline EG parto & $-0,12$ & $-0,24$ a $-0,00$ & 0,04 & 257 \\
\hline Plaquetas & $-0,04$ & $-0,20$ a 0,11 & 0,57 & 145 \\
\hline Acido Úrico & 0,11 & $-0,10$ a 0,32 & 0,29 & 85 \\
\hline
\end{tabular}

Correlación de Pearson, r. Intervalo de confianza del 95\%, 95\% IC. Presión arterial sistólica, PAS. Presión arterial diastólica, PAD. Recién nacido, RN. Edad gestacional, EG. 
Tabla VI

RELACION DEL NUMERO DE PLAQUETAS Y LOS NIVELES DE ACIDO URICO CON VARIABLES CLINICAS MATERNAS Y NEONATALES EN LAS MUJERES CON SINDROME HIPERTENSIVO DEL EMBARAZO EN EL HOSPITAL HERMINDA MARTIN DE CHILLAN

\begin{tabular}{|c|c|c|c|c|}
\hline Variable & $r$ & $95 \%$ IC & $p$ & $\mathrm{n}$ \\
\hline \multicolumn{5}{|c|}{ Número de Plaquetas } \\
\hline Peso RN & 0,21 & 0,04 a 0,36 & 0,01 & 137 \\
\hline Percentil peso RN & 0,08 & $-0,08$ a 0,24 & 0,34 & 137 \\
\hline Talla RN & 0,17 & 0,00 a 0,33 & 0,05 & 135 \\
\hline Percentil talla RN & 0,12 & $-0,04$ a 0,28 & 0,15 & 135 \\
\hline EG parto & $-0,009$ & $-0,17$ a 0,15 & 0,91 & 143 \\
\hline Acido Úrico & $-0,07$ & $-0,29$ a 0,15 & 0,52 & 75 \\
\hline \multicolumn{5}{|c|}{ Niveles de Acido Úrico } \\
\hline Peso RN & $-0,26$ & $-0,45$ a $-0,05$ & 0,01 & 82 \\
\hline Percentil peso RN & $-0,30$ & $-0,49$ a $-0,09$ & 0,005 & 82 \\
\hline Talla RN & $-0,28$ & $-0,47$ a $-0,07$ & 0,009 & 82 \\
\hline Percentil talla RN & $-0,30$ & $-0,49$ a $-0,01$ & 0,005 & 82 \\
\hline EG parto & $-0,17$ & $-0,37$ a 0,04 & 0,11 & 85 \\
\hline
\end{tabular}

Correlación de Pearson, r. Intervalo de confianza del 95\%, 95\% IC. Presión arterial sistólica, PAS. Presión arterial diastólica, PAD. Recién nacido, RN. Edad gestacional, EG.

Tabla VII

ACIDO URICO Y VARIABLES CLINICAS MATERNAS Y NEONATALES EN LAS MUJERES CON SINDROME HIPERTENSIVO DEL EMBARAZO EN EL HOSPITAL HERMINDA MARTIN DE CHILLAN

\begin{tabular}{lcccc}
\hline Característica & $\begin{array}{c}\text { Bajo } \\
(\mathrm{n}=26)\end{array}$ & $\begin{array}{c}\text { Medio } \\
(\mathrm{n}=38)\end{array}$ & $\begin{array}{c}\text { Alto } \\
(\mathrm{n}=19)\end{array}$ & $\mathrm{p}$ \\
\hline IMC materno & $36,8 \pm 1,7$ & $36,2 \pm 1,1$ & $33,1 \pm 1,1$ & $\mathrm{NS}$ \\
PAS & $143,8 \pm 2,6$ & $148,3 \pm 3,1$ & $152,7 \pm 4,3$ & $\mathrm{NS}$ \\
PAD & $92,7 \pm 2,3$ & $97,7 \pm 2,7$ & $98,8 \pm 2,8$ & $\mathrm{NS}$ \\
EG parto & $273,6 \pm 1,6$ & $265,7 \pm 2,2$ & $257,8 \pm 3,8^{*}$ & 0,002 \\
Peso RN (g) & $3545 \pm 123$ & $3279 \pm 111$ & $2761 \pm 143^{*} \ddagger$ & 0,0007 \\
Percentil peso RN & $64,5 \pm 5,9$ & $63,2 \pm 4,2$ & $45,2 \pm 6,6^{*}$ & 0,04 \\
Talla RN (g) & $49,8 \pm 0,3$ & $48,7 \pm 0,4$ & $46,6 \pm 0,7^{*} \ddagger$ & 0,001 \\
Percentil Talla RN & $60,0 \pm 5,1$ & $57,9 \pm 4,4$ & $42,3 \pm 6,3$ & 0,05 \\
\hline
\end{tabular}

Valores expresados en promedio \pm error estándar (E.E). Presión arterial sistólica, PAS. Presión arterial diastólica, PAD. Recién Nacido, RN. Bajo, ácido úrico < percentil 25. Medio, ácido úrico entre el percentil 25 y 75 . Alto, ácido úrico superior al percentil 75 de acuerdo a la distribución de los valores en la población estudiada. ${ }^{\star} \mathrm{p}<0,05$ vs Bajo. $¥ \mathrm{p}<0,05$ vs Medio. No estadísticamente significativo, NS 


\section{DISCUSIÓN}

Los resultados encontrados muestran una prevalencia de SHE en el hospital Herminda Martín de Chillán de 3,8\% entre el 2006-2008. Se encontraron diferencias importantes en los criterios diagnósticos utilizados en este centro de salud respecto a las normas a nivel nacional. Al estratificar las mujeres con SHE, se encontró que las mujeres con presión arterial en el rango de pre-eclampsia moderada y severa muestran características clínicas y neonatales similares a otras publicaciones nacionales $(12,20,21)$. El IMC, la presión arterial materna y el nivel de ácido úrico, están relacionados negativamente con la antropometría neonatal. Además, la antropometría neonatal fue menor en las mujeres con niveles más altos de ácido úrico, situación que no obedecería a la severidad de la hipertensión o el IMC materno.

En este estudio se encontró una tasa de SHE $(3,8 \%)$ apreciablemente menor que en el contexto nacional. Sin embargo, claramente esta cifra puede cambiar dado que del total de historias revisadas, un número considerable de mujeres no cumplían los criterios clínicos de presión arterial para ser catalogadas como hipertensas (Grupo I, n=124). Además, específicamente el diagnóstico de preeclampsia fue posible determinar en un pequeño grupo de mujeres que cumplían criterios de hipertensión y proteinuria. Por ello, la utilización de los criterios diagnósticos en el Hospital de Chillán, se alejan de las recomendaciones a nivel nacional, y se asemejan a los entregados por la Sociedad Australiana de Estudio de la Hipertensión del Embarazo (6). Por consiguiente, estas situaciones enmascaran la prevalencia de SHE y particularmente de preeclampsia en dicho hospital. Actualmente, la Dirección del Departamento de Ginecología y Obstetricia, esta mejorando y estandarizando los criterios diagnósticos en diversas patologías y en particular del SHE.

Las características clínicas como edad, nuliparidad, y antecedentes de hipertensión en las mujeres con SHE moderada (Grupo II) y severa (Grupo III), fueron similares a otros estudios nacionales $(12,20,21)$. Además, en nuestro análisis $\sim 60 \%$ de las mujeres con SHE tuvieron obesidad, situación que ha sido mostrada en otros estudios internacionales $(22,23)$. Si bien este aumento de peso (reflejado en el IMC) pudiera obedecer a retención de líquidos y edema presente en las embarazadas con hipertensión, resulta importante considerar que la obesidad en el embarazo se relaciona con otras condiciones patológicas como aborto espontáneo, aborto recurrente, diabetes gestacional, defectos del tubo neural (24) e insulino-resistencia fetal (25).
Esto último es alarmante, dado que de acuerdo a la hipótesis de programación fetal los insultos recibidos en la etapa gestacional predisponen al neonato a sufrir de enfermedades crónicas en su vida adulta. Por ello, el control de peso durante la gestación es una estrategia a ser considerada y mejorada en el Hospital de Chillán.

Es interesante resaltar los casos de mujeres con SHE severa (Grupo III), en cuyo grupo están concentradas las mujeres con obesidad, que tuvieron hijos de bajo peso. Si bien en nuestro estudio existió una correlación positiva entre IMC materno y peso del recién nacido (ajustado o no para la edad gestacional), es probable que la suma de las patologías presentes en las madres (obesidad e hipertensión) lleve a una reducción del aporte sanguíneo hacia el feto predisponiéndolo a bajo peso. En este sentido, se conoce que a un IMC superior a 30 , la relación entre IMC materno y peso del recién nacido es inversa (24). Adicionalmente, encontramos que el IMC materno se relacionó con el nivel de ácido úrico, como fuera mostrado por otro grupo (26). Por ello, es posible especular que la combinación de estos marcadores (hipertensión, obesidad y ácido úrico) podría servir como cribado para el bienestar fetal.

La edad gestacional, peso y talla del recién nacido encontrados en este estudio fueron similares a los encontrados en otros a nivel nacional $(12,21)$. Es conocido que las mujeres con SHE tienen mayor riesgo de parto prematuro $(1,8)$, situación que se confirma en el presente estudio al analizar la edad gestacional de parto y el porcentaje de partos prematuros (<35 semanas) en mujeres con SHE severa (Grupo III). Además, existe una correlación inversa entre la presión arterial sistólica y diastólica y la edad gestacional de parto. En este sentido, se conoce que la falta de un tratamiento dirigido a la(s) causa(s) de la preeclampsia, induce a una terminación pronta del embarazo como única estrategia que revierte la condición clínica $(1,27)$. Sin embargo, esta acción no esta exenta de riesgo, ya que el parto prematuro conlleva a mayor morbi-mortalidad neonatal y fetal que agrava el cuadro clínico de preeclampsia $(1,8)$. Precisamente, la tasa de mortalidad fetal en este grupo de estudio fue de $3,4 \%$, lo que significó una relación de mortalidad fetal (número de muertes fetales/número de nacidos vivos) de 0,83 x 1000 nacidos vivos, una tasa inferior a las estadísticas nacionales que evidentemente excluye otras causas de mortalidad fetal y que probablemente constituya un subregistro dado que no se pudo revisar el universo de pacientes con SHE en el periodo entre el 2006 a 2008. Sin embargo, de los datos 
disponibles es notorio el hecho que precisamente las muertes fetales se encontraron en los grupos de pacientes con rangos de presión arterial bajos (Grupo I) o moderados (Grupo II), mientras que no se tuvieron casos de mortalidad fetal en los casos de hipertensión severa (Grupo III). Estos resultados se contraponen a estudios nacionales (12) o internacionales (1), y podrían estar asociados a la decisión urgente de terminar el embarazo en casos de SHE severa por el equipo clínico (mayor número de partos <35 semanas en este grupo), o que la mortalidad en este grupo de embarazos supere la etapa neonatal (28 días), entre otras.

Adicionalmente, el peso y la talla del recién nacido fue menor en las mujeres con hipertensión severa, situación que no obedecería a las diferencias en la edad gestacional de parto. Estos hallazgos justifican el mayor porcentaje de diagnóstico de RCIU en las mujeres con hipertensión severa en nuestro estudio, corroborando también estadísticas internacionales $(1,8,28,29)$ y nacionales $(12,14)$. Además, la presión arterial sistólica o diastólica se correlacionó inversamente con el peso del recién nacido, sugiriendo que la reducción en el aporte sanguíneo placentario, producto de la vasoconstricción materna generalizada y en forma particular en las arterias uterinas, podría estar involucrado en la reducción del crecimiento fetal (30).

Es importante resaltar, que en la muestra estudiada no se encontraron registros de casos compatibles con síndrome de HELLP, cuya aparición ha sido anecdótica en el hospital (15). Adicionalmente, la falta de diferencias significativas en los parámetros bioquímicos analizados de las mujeres con SHE respecto a la función hepática, renal, o hemólisis, puede obedecer al número de casos analizados (aproximadamente 90), o al hecho que en nuestro estudio utilizamos únicamente el nivel de presión arterial para clasificar las pacientes, entre otras.

El conteo plaquetario fue significativamente menor en las pacientes con hipertensión severa. Este hallazgo ha sido asociado a un aumento en el recambio plaquetario subsiguiente a activación en la circulación materna $(31,32)$. Adicionalmente, el número de plaquetas en la muestra analizada se correlacionó positivamente con el peso y la talla del recién nacido, corroborando resultados previamente mostrados por otro grupo (33). Por otro lado, la trombocitopenia materna en mujeres con preeclampsia se asocia también a trombocitopenia neonatal (33). Por lo dicho, es posible sugerir que el número de plaquetas en las mujeres con hipertensión arterial durante el embarazo, podría ser más que un marcador de riesgo materno. De hecho, se conoce que la adecuada función plaquetaria podría estar participando en la regulación del flujo placentario (34) y por intermedio de ello probablemente en el crecimiento fetal.

El nivel de ácido úrico se correlacionó negativamente con el peso y la talla de los recién nacidos en forma independiente de la edad gestacional de parto. Además, las mujeres hipertensas con un nivel de ácido úrico alto (>p75), tuvieron hijos de menor peso y talla, y partos de menor edad gestacional que las mujeres con niveles de ácido úrico bajos $(<$ p25) o medios (p25-75). Estos hallazgos se integran con otros estudios internacionales $(7,35,36)$, en donde el nivel de ácido úrico se utilizó para caracterizar a mujeres con pre-eclampsia que presentan alto riesgo de morbilidad y mortalidad materna y fetal. Particularmente, los niveles de ácido úrico elevados en mujeres con pre-eclampsia están asociados a parto pretérmino y RCIU (7). Precisamente, estas evidencias han llevado a proponer un rol patogénico del ácido úrico en las complicaciones fetales (37), particularmente relacionado con crecimiento fetal y el transporte de nutrientes (aminoácidos) a través de la placenta (38), inhibición del proceso de placentación (39) y como marcador de disfunción endotelial en la placenta $(39,40)$. Pese a estas evidencias, los mecanismos fisiopatológicos de la asociación entre ácido úrico y mayor riesgo perinatal en embarazos con hipertensión u otros embarazos patológicos $(41,42)$ todavía están bajo estudio.

Finalmente reconocemos la limitación de este estudio retrospectivo, en donde la información disponible no abarcó el universo de pacientes con SHE en el periodo estudiado. Por lo que es necesaria la realización de estudios prospectivos que permitan estimar el apego a los criterios diagnósticos, las características del manejo clínico y la incidencia real de complicaciones maternas y neonatales en mujeres con SHE en este hospital.

\section{CONCLUSIÓN}

Se determinó que para el diagnóstico de SHE en el Hospital Clínico Herminda Martín de Chillán (2006-2008) se desestimó la utilización de la proteinuria. La presencia de SHE esta asociada a mayor morbilidad materna y neonatal como ha sido mostrado en otros estudios nacionales. Este estudio permitió detectar debilidades del Servicio y hacer recomendaciones sobre probables marcadores de riesgo perinatal (IMC materno, plaquetas, ácido úri$\mathrm{co})$. 
Agradecimientos. Al Dr. Rodrigo Avendaño, Director del Hospital Herminda Martin y por su intermedio al personal administrativo y de estadística por facilitar la revisión de las fichas clínicas. Este trabajo fue financiado por la Dirección de Investigaciones, Universidad del Bío-Bío, proyecto 0965091/RS y el Fondo Nacional de Desarrollo Científico y Tecnológico proyecto FONDECYT 1100684.

\section{BIBLIOGRAFÍA}

1. Sibai B, Dekker G, Kupferminc M. Preeclampsia. Lancet 2005;365(9461):785-99.

2. ACOG Committee on Practice Bulletins--Obstetrics. ACOG practice bulletin. Diagnosis and management of preeclampsia and eclampsia. Number 33, January 2002. Obstet Gynecol 2002; 99(1):159-67.

3. Roberts JM, Gammill HS. Preeclampsia: recent insights. Hypertension 2005;46(6):1243-9.

4. Duley L, Meher S, Abalos E. Management of preeclampsia. BMJ 2006;332(7539):463-8.

5. Sibai BM, Barton JR. Expectant management of severe preeclampsia remote from term: patient selection, treatment, and delivery indications. Am J Obstet Gynecol 2007;196(6):514.e1-9.

6. Thornton CE, Makris A, Ogle RF, Tooher JM, Hennessy $A$. The role of proteinuria in defining preeclampsia - clinical outcomes for women and babies. Clin Exp Pharmacol Physiol 2010;37(4):466-70

7. Roberts JM, Bodnar LM, Lain KY, Hubel CA, Markovic $\mathrm{N}$, Ness RB, Powers RW. Uric acid is as important as proteinuria in identifying fetal risk in women with gestational hypertension. Hypertension 2005;46(6):12639.

8. Villar J, Carroli G, Wojdyla D, Abalos E, Giordano D, Ba'aqeel $\mathrm{H}$, et al. Preeclampsia, gestational hypertension and intrauterine growth restriction, related or independent conditions?. Am J Obstet Gynecol 2006;194(4):921-31.

9. Teran E, Escudero C, Vivero S, Molina G, Calle A. NO in early pregnancy and development of preeclampsia. Hypertension 2006;47(4):e17.

10. Duley L. The global impact of preeclampsia and eclampsia. Semin Perinatol 2009;33(3):130-7.

11. Pedrasa D, Silva A. Sindrome hipertensivo del embarazo. En: Salinas H, Parra M, Valdés E, Carmona S, Opazo D. Editores. Guias Clínicas. Departamento de Obstetricia y Ginecología. Hospital Clínico Universidad de Chile. 2005: 329-36.

12. Parra M, San Martín A, Valdés E, Quiroz L, Schepeler $M$, Pérez $S$, et al. Espectro clínico de la preeclampsia: estudio comparativo de los distintos grados de severidad. Rev Chil Obstet Ginecol 2007;72(3):169-75.

13. Donoso $\mathrm{E}$. ¿De qué mueren las madres chilenas en el siglo XXI? Cambios del perfil epidemiológico. Rev Chil Obstet Ginecol 2009;74(4):2007-8.

14. Molina M, Casanueva V, Pérez R, Ferrada C, Cisternas J, Cid L, et al. [Impact of hypertensive disease of pregnancy on intrauterine growth retardation]. Rev Med Chil 1998;126(4):375-82.

15. Ribbeck G, Muñoz C, Gutiérrez S. Mola incompleta, eclampsia y síndrome de HELLP: un caso clínico. Rev Chil Ultrasonog 2006;9(2):62-6.
16. Muñoz $M$, Oliva $P$. Los estresores psicosociales se asocian a síndrome hipertensivo del embarazo y/o síntomas de parto prematuro en el embarazo adolescente. Rev Chil Obstet Ginecol 2009;74(5):281-5.

17. Ministerio de Salud. Departamento de estadísticas e información de salud. 2005 Hallado en: http://163.247.51.54/EGRESOS1/egreso2005/mujeres/egre_causa.php

18. Hadad B, Barton JR, Livingston JC, Chahine R, Sibai BM. HELLP (hemolysis, elevated liver enzymes, and low platelet count) syndrome versus severe preeclampsia: Onset at 28.0 weeks of gestation. Am J Obstet Gynecol 2000;183 (6):1475-9.

19. González RP, Gómez RM, Castro RS, Nien JK, Merino $P O$, Etchegaray $A B$, et al. [A national birth weight distribution curve according to gestational age in Chile from 1993 to 2000]. Rev Med Chil 2004;132(10):115565.

20. Chamy V, Madrid E, Aránguiz N, Guerra V, Cárcamo $\mathrm{K}$, Rejas A. Perfil clínico de embarazadas con preeclampsia y embarazos no complicados. Rev Chil Obstet Ginecol 2004;69(5): 361-7.

21. Parra $M$, Rodrigo $R$, Barja $P$, Bosco $C$, Fernández $\mathrm{V}$, Muñoz $\mathrm{H}$, et al. Screening test for preeclampsia through assessment of uteroplacental blood flow and biochemical markers of oxidative stress and endothelial dysfunction. Am J Obstet Gynecol 2005;193(4):1486-91.

22. Madan J, Chen M, Goodman E, Davis J, Allan W, Dammann $O$. Maternal obesity, gestational hypertension, and preterm delivery. J Matern Fetal Neonatal Med 2010;23(1):82-8.

23. Crane JM, White J, Murphy P, Burrage L, Hutchens $D$. The effect of gestational weight gain by body mass index on maternal and neonatal outcomes. J Obstet Gynaecol Can 2009;31(1):28-35.

24. Leddy MA, Power ML, Schulkin J. The impact of maternal obesity on maternal and fetal health. Rev Obstet Gynecol 2008;1(4):170-8.

25. Catalano PM, Presley L, Minium J, Hauguel-de Mouzon S. Fetuses of obese mothers develop insulin resistance in utero. Diabetes Care 2009;32(6):107680.

26. Rajasingam D, Seed PT, Briley AL, Shennan AH, Poston L. A prospective study of pregnancy outcome and biomarkers of oxidative stress in nulliparous obese women. Am J Obstet Gynecol 2009;200(4):395.e1-e9.

27. Basso O, Rasmussen S, Weinberg CR, Wilcox AJ, Irgens LM, Skjaerven $R$. Trends in fetal and infant survival following preeclampsia. JAMA 2006;296(11):135762.

28. Buchbinder A, Sibai BM, Caritis S, Macpherson C, Hauth J, Lindheimer MD, et al; National Institute of Child Health and Human Development Network of Maternal-Fetal Medicine Units. Adverse perinatal outcomes are significantly higher in severe gestational hypertension than in mild preeclampsia. Am J Obstet Gynecol 2002;186(1):66-71.

29. Xiong X, Buekens P, Pridjian G, Fraser WD. Pregnancy-induced hypertension and perinatal mortality. J Reprod Med 2007;52(5):402-6.

30. Friedman EA, Neff RK. Hypertension-hypotension 
in pregnancy. Correlation with fetal outcome. JAMA 1978;239(21):2249-51.

31. Romero R, Duffy TP. Platelet disorders in pregnancy. Clin Perinatol 1980;7(2):327-48.

32. Dundar O, Yoruk P, Tutuncu L, Erikci AA, Muhcu M, Ergur AR, et al. Longitudinal study of platelet size changes in gestation and predictive power of elevated MPV in development of pre-eclampsia. Prenat Diagn 2008; 28(11): 1052-1056.

33. Bhat YR, Cherian CS. Neonatal thrombocytopenia associated with maternal pregnancy induced hypertension. Indian J Pediatr 2008;75(6):571-3.

34. Tsao PN, Wei SC, Su YN, Chou HC, Chen CY, Hsieh WS. Excess soluble fms-like tyrosine kinase 1 and low platelet counts in premature neonates of preeclamptic mothers. Pediatrics 2005;116(2):468-72.

35. Thangaratinam S, Ismail KM, Sharp S, Coomarasamy A, Khan KS.Tests in prdiction of Pre-eclampsia Severity review group. Accuracy of serum uric acid in predicting complications of pre-eclampsia: a systematic review. BJOG 2006; 113(4): 369-78.

36. Koopmans CM, van Pampus MG, Groen H, Aarnoudse JG, van den Berg PP, Mol BW. Accuracy of serum uric acid as a predictive test for maternal complications in pre-eclampsia: bivariate meta-analysis and decision analysis. Eur J Obstet Gynecol Reprod Biol 2009;146(1):8-14.

37. Bainbridge SA, Roberts JM. Uric acid as a pathogenic factor in preeclampsia. Placenta 2008;29(Suppl A):S67-S72.

38. Bainbridge SA, von Versen-Höynck F, Roberts JM. Uric acid inhibits placental system amino acid uptake. Placenta 2009;30(2):195-200.

39. Bainbridge SA, Roberts JM, von Versen-Höynck F, Koch J, Edmunds L, Hubel CA. Uric acid attenuates trophoblast invasion and integration into endothelial cell monolayers. Am J Physiol Cell Physiol 2009;297(2):C440-C50.

40. Anceschi MM, Piazze JJ, Maranghi L, Ruozi-Berretta A, Cosmi EV. Maternal serum uric acid as predictor of fetal acidemia. Int J Gynaecol Obstet 2002;77(1):35-6.

41. Laughon SK, Catov J, Provins T, Roberts JM, Gandley RE. Elevated first-trimester uric acid concentrations are associated with the development of gestational diabetes. Am J Obstet Gynecol 2009;201(4):402.e1e5.

42. Franco MC, Christofalo DM, Sawaya AL, Ajzen SA, Sesso R. Effects of low birth weight in 8- to 13-yearold children: implications in endothelial function and uric acid levels. Hypertension 2006;48(1):45-50. 